\title{
Pathology of hyperextension injury of the cervical spine: a case report
}

\author{
H Kinoshita
}

Director, Kosei Hospital, Hiroshima, Japan

Keywords: pathology; cervical spine; hyperextension injury

A 61-year-old man whilst intoxicated fell into a deep ditch and suffered a hyperextension injury at $\mathrm{C} 5 / 6$ level. There was loss of sensation and motor control below the level of injury. X-rays of the neck showed that there was no fracture or disc injury. He died 17 months after the injury from bronchopneumonia.

Postmortem was performed immediately after death, and the spinous processes and neural arches were cut longitudinally long the midline and the spinal

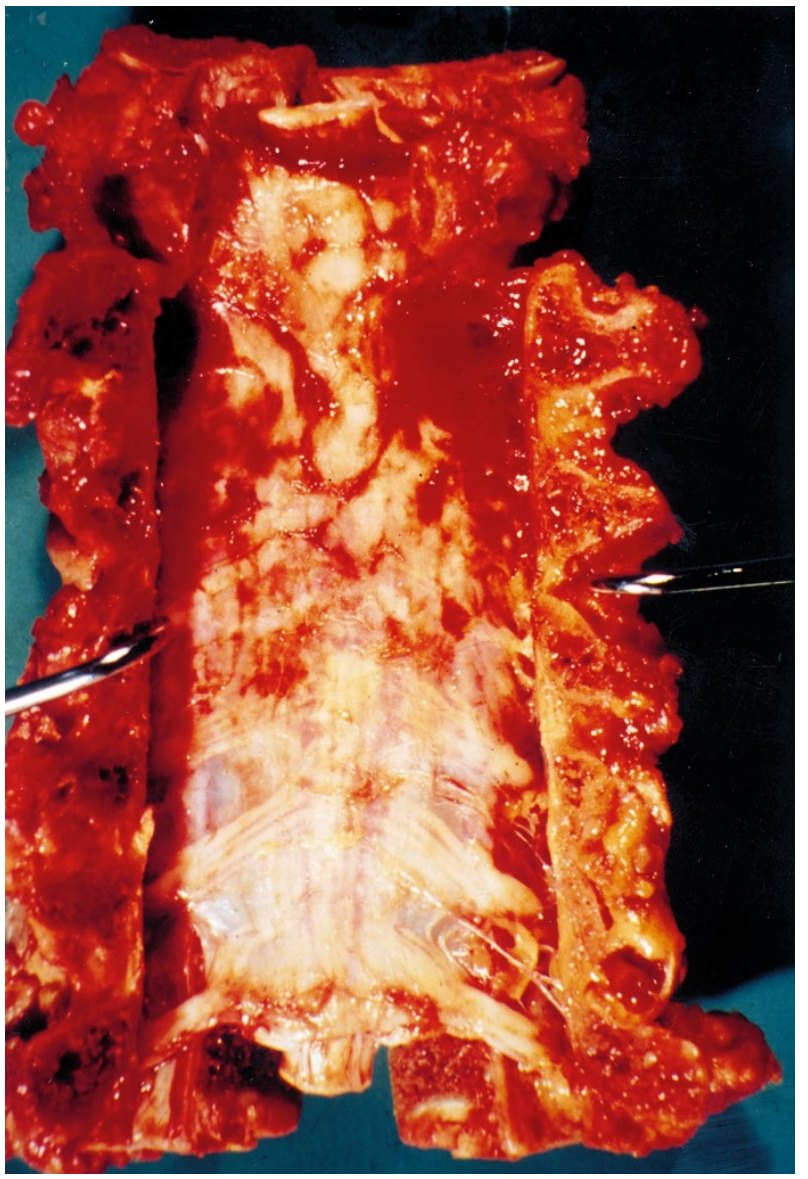

Figure 1 Vertebral bodies were cut and spinal canal was widely opened. Extradural haemorrhage was observed from $\mathrm{C} 1$ to $\mathrm{C} 4$ canal was opened widely from behind. The extradural and intradural spaces including the spinal cord, spinal nerve roots, dentate ligaments and leptomeninges were carefully observed grossly, and this revealed an extradural haemorrhage from $\mathrm{C} 1$ to $\mathrm{C} 4$ (Figure 1), and attenuation of the nerve roots of $\mathrm{C} 3,4$ and 5 (Figure 2). The anterior parts of the bodies from $\mathrm{C} 2$ and 6 were fused. Spinal stenosis was not present although a bony protuberance $5 \mathrm{~mm}$ in diameter was

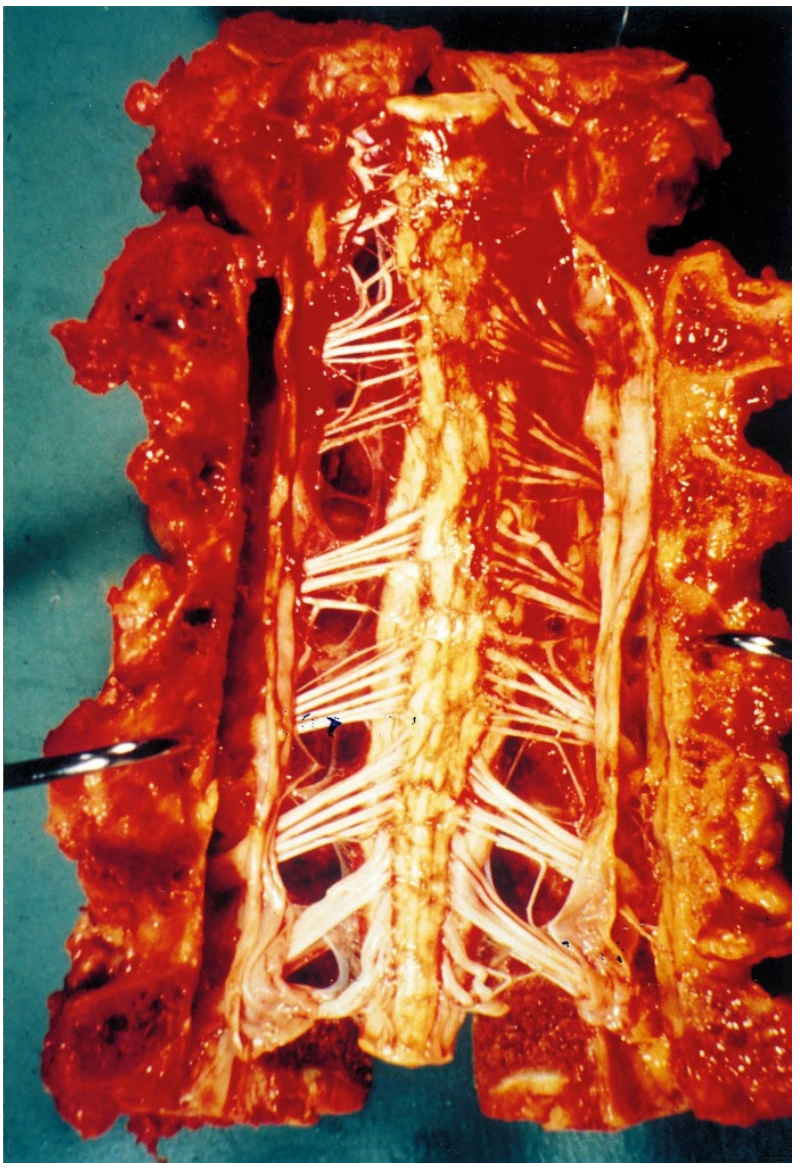

Figure 2 Spinal cord and spinal nerve roots were removed. Attenuation of nerve roots of $\mathrm{C} 3,4$ and 5 with blood staining could be observed 


Cervical spinal cord injury
$\mathrm{H}$ Kinoshita et al

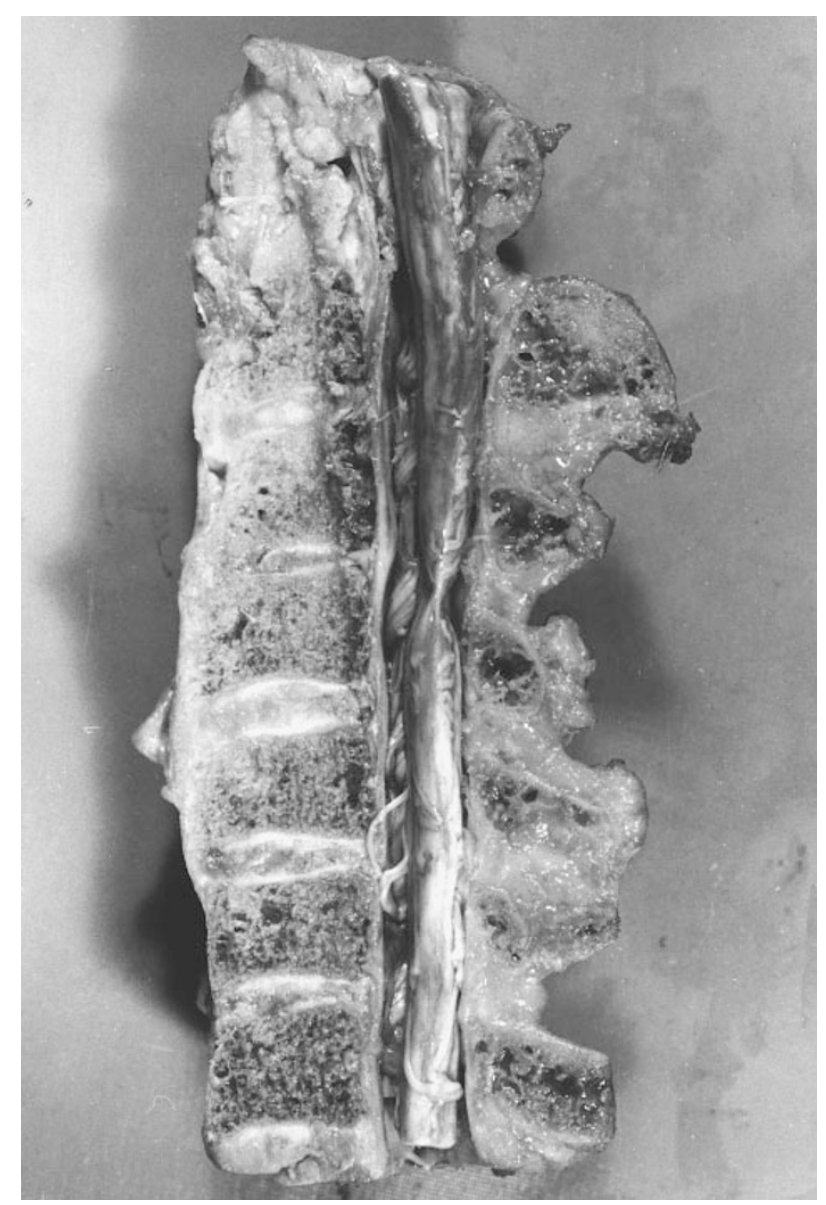

Figure 3 Sagittal section of the spine. Spinal stenosis was not present, although a bony protuberance $5 \mathrm{~mm}$ in diameter was present behind the body of $\mathrm{C} 4$ present behind the body of $\mathrm{C} 4$ (Figure 3). It is presumed that this osteophyte compressed the spinal cord as a result of a hyperextension injury. Cross sections of the spinal cord showed total absence of parenchyma at the level of injury. 